\title{
Estudio de prevalencia del consumo de drogas en escolares de Enseñanza Básica, a través de la metodología de pares
}

\author{
Jorge Rodríguez ${ }^{1 a}$, Ana María Fernández ${ }^{2 b}$, \\ Enrique Hernández ${ }^{1 c}$, Macarena Valdés ${ }^{3 d}$,' \\ Marcelo Villalón ${ }^{1}$, Sergio Ramírez ${ }^{4}$, Rafael Ramíreze, \\ Catalina Valenzuela ${ }^{4}$, Sebastián Cardemil ${ }^{4}$. \\ Prevalence of drug abuse among \\ children in basic schools
}

Background: Peer methodology (PM) is an adaptation of the privileged access interviewer methodology and is used for prevalence studies. PM estimates higher drug consumption frequencies among school and college students than self reports, since it minimizes underreporting. Aim: To assess drug abuse among students using PM. Material and methods: A random sample of 234 school students of middle and low-middle socioeconomic status, aged 9 to 14 years (53\% women) were interviewed using PM about drug consumption. Results: The frequency of licit drug consumption during the last year was $29 \%$ and $15 \%$ during the last month (current consumption). The consumption of tobacco and alcohol was $20 \%$ and $18 \%$ respectively during the last year. The figures for the last month were $11 \%$ and $8 \%$ respectively. The beginning age for smoking and alcohol consumption were $11.1 \pm 1.5$ and $11.3 \pm 1.8$ years respectively. The frequency of illicit drug consumption was 3.5\%. Illicit drug users began licit drug consumption at earlier ages their non user peers. Conclusions: There is a high frequency of illicit drug consumption among school age children (Rev Méd Chile 2009; 137: 774-84).

(Key w ords: Children; Street drugs; Substance abuse detection)
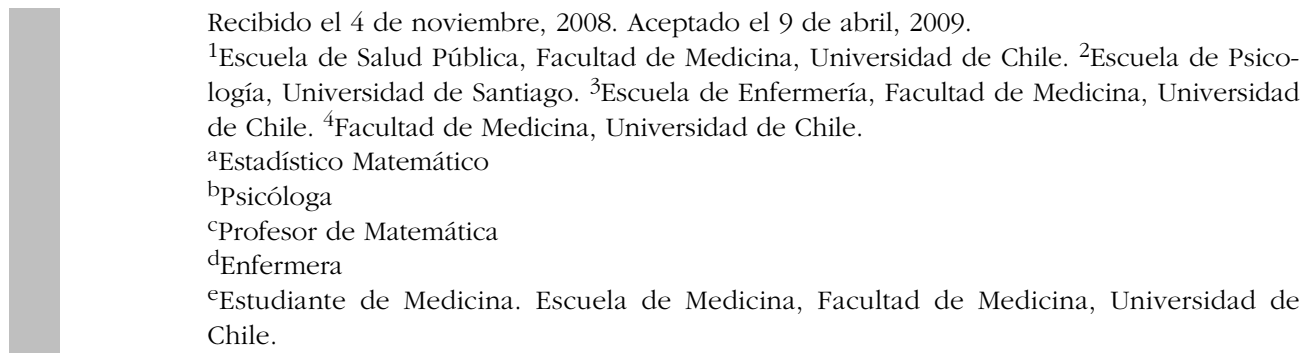
Chile.

$\overline{\text { Correspondencia a: Jorge Rodríguez T. Independencia } 939 .}$

Santiago, Chile. Fax: 7774163.

E mail : jrodrigu@med.uchile.cl 
$M$ uchos países se han visto afectados por el consumo creciente de drogas lícitas e ilícitas, entre ellas destacan: alcohol, marihuana, clorhidrato de cocaína, pasta base y éxtasis. Dicho problema impacta con mayor fuerza a jóvenes, observándose que cada día, más adolescentes inician el consumo de drogas y muchos de ellos continúan el consumo en forma recurrente, llevando a un aumento progresivo de las tasas de drogadicción ${ }^{1-4}$.

Diversos estudios internacionales describen la problemática del consumo de drogas en adolescentes y sus consecuencias para la sociedad. Un estudio realizado en adolescentes de Tailandia 5 encontró una prevalencia de consumo de cigarrillos de 5,4\%, de alcohol de 37,3\% y de 37,8\% para drogas ilícitas. Otro estudio en universitarios de Sao Paulo ${ }^{6}$, señala el aumento del consumo de drogas lícitas en mujeres adolescentes, sin embargo, los varones presentan mayor tendencia al consumo excesivo (86,5\% para el tabaco y 65\% para el alcohol $)^{6}$. En una universidad irlandesa ${ }^{7}$, un estudio prospectivo (1973-2002) evaluó la evolución del consumo de drogas en estudiantes de medicina, evidenciando una disminución del consumo de cigarrillos (de $28,8 \%$ a 9,2\%), en contraste con una fuerte alza del consumo de alcohol.

El uso y abuso de drogas afecta la salud y se asocia con conductas de violencia y victimización, afectando la estabilidad de todas las instituciones que conforman la sociedad $2,3,5,6,8-10$. En un estudio en universitarios chilenos ${ }^{8}, 21 \%$ había participado en riñas o peleas durante el último año y 11,3\% lo había hecho en los últimos 30 días, se observó que el subgrupo de estudiantes con conductas de violencia presentaba una mayor prevalencia, aunque no significativa, de consumo reciente y actual de tabaco y de alcohol, y una prevalencia significativamente mayor de consumo reciente y actual de marihuana, cocaína y éxtasis, respecto de aquellos estudiantes sin antecedentes de violencia.

En Chile, el CONACE (Consejo Nacional para el Control de Estupefacientes) ha realizado múltiples estudios para estimar la magnitud del consumo de drogas lícitas e ilícitas en población escolar y general ${ }^{11,12}$. Los últimos cuatro estudios realizados en población escolar de octavo básico a cuarto medio, permiten esbozar la evolución cronológica del problema a pesar de tratarse de estudios de corte transversal. El último estudio en escolares (2007) mostró una elevada prevalencia de consumo reciente y actual de alcohol y de tabaco. La prevalencia de abuso de alcohol (5 vasos o más en una salida habitual) se mantuvo estable en $12 \%$ y su uso intensivo (10 o más días en los últimos 30 días) aumentó de 7,9\% a 9\%. También mostró un alza en el consumo actual de marihuana (desde 16,6\% a 20,4\%), cocaína (4,4\% a $5,6 \%)$, pasta base $(3,9 \%$ a $4,9 \%)$ y éxtasis $(3,4 \%$ a $3,9 \%$ ). Igualmente alarmante fue la creciente prevalencia de creencias que favorecen el consumo de marihuana (pensar que no es adictiva, que su uso experimental no provoca daño y que la mayoría de los jóvenes la consume) y la disminución de la percepción de riesgo, disminuyendo desde $40 \%$ a 30\% el porcentaje de los escolares que considera riesgoso el uso experimental de marihuana (probarla 1 ó 2 veces) y desde 52\% a $39 \%$ los que consideran riesgoso su uso frecuente (2 o más veces/semana).

Si bien los estudios mencionados permiten esbozar el problema del consumo de drogas ilícitas por los escolares de nuestro país, no permiten estimar adecuadamente la magnitud del mismo, ya que los encuestados evitan declarar el consumo de drogas por temor a ser discriminados o sancionados, llevando a subvalorar el problema. Las "poblaciones ocultas" (hidden population) ${ }^{13}$ son grupos de individuos que no son representados por los estudios poblacionales clásicos, ya que son "invisibles" a los sistemas de recolección de datos usados habitualmente para realizar estimaciones poblacionales. Se trata de individuos que presentan características de baja aceptación social, como la drogadicción, delincuencia, abuso sexual, maltrato infantil, etc., de modo que portan dicho rasgo en silencio.

Existen metodologías especiales para evaluar "poblaciones ocultas", siendo una de las más conocidas la de "Entrevistadores de Acceso Privilegiado" (EAP) ${ }^{14-18}$. A comienzos de 2000 surgió en Chile la "Metodología de Pares" desarrollada como una adaptación de la metodología de EAP, a los estudios de prevalencia, demostrando ser efectiva para disminuir la subdeclaración del consumo de drogas ${ }^{19-21}$. En un estudio con Metodología de Pares en universitarios chilenos de 18-26 años ${ }^{20}$, 63,4\% declaró consumir marihuana, 4,9\% cocaína y 2,1\% éxtasis, obteniendo 
índices mayores a los observados en estudios con metodologías tradicionales. El sexo femenino mostró índices inferiores, pero no significativos, de consumo de alcohol y de drogas ilícitas respecto de los varones y un consumo de tabaco significativamente mayor, con valores superiores a los reportados por estudios con metodologías tradicionales ${ }^{22}$.

Por lo tanto, al problema del consumo de drogas debemos añadir el problema de la subdeclaración, pues habría un subgrupo altamente vulnerable a la drogadicción y a las conductas de riesgo asociadas que no está siendo abordado por las estrategias de prevención y promoción de la salud. De lo anterior, podemos preguntarnos: ¿Cuál es la magnitud de consumo en población escolar básica?, ¿los estudios están identificando a los grupos de riesgo o aquellos que ya tienen la conducta?, ¿se está interviniendo oportunamente para prevenir el consumo?

El objetivo de esta investigación fue estimar con mayor precisión la magnitud del consumo de drogas en escolares, como también evaluando posibles asociaciones con variables sociodemográficas (género, composición familiar, conductas de riesgo), usando la Metodología de Pares e incluyendo a escolares con edades consideradas tradicionalmente de riesgo para el consumo de drogas.

\section{MATERIAL Y MÉTODO}

El universo de estudio estuvo comprendido por escolares de 9 a 14 años provenientes de colegios municipalizados o subvencionados de las comunas de Recoleta y Huechuraba, perteneciendo a niveles socioeconómicos medio, medio-bajo y bajo.

El tamaño muestral mínimo se calculó en 216 escolares, considerando una tasa de consumo de drogas lícitas de 10\%, un nivel de confianza de $95 \%$, un error máximo de estimación de $4 \%$ y una potencia de $50 \%$. Dos colegios accedieron a participar en el estudio (uno municipalizado y uno subvencionado) dando las facilidades necesarias para utilizar la Metodología de Pares, la cual involucra seleccionar y capacitar alumnos voluntarios del establecimiento para entrevistar al resto del alumnado ${ }^{19,20}$. Se supuso un comportamiento (consumo de drogas) heterogéneo de los entrevistados al interior de cada colegio y homogéneo entre colegios.

Para recoger la información se utilizó un instrumento previamente adaptado para este grupo de escolares, a partir de uno utilizado que fue validado en población universitaria ${ }^{23}$, la adaptación consistió en una validación de contenido por tres jueces, que sugirieron modificar y eliminar aquellas preguntas que consideraron no apropiadas para este grupo etáreo (Anexo I y II). Se consideró como drogas lícitas al tabaco y el alcohol, definiendo como consumidores de tabaco a quienes fuman al menos dos veces por semana y como consumidores de alcohol a quienes beben al menos una vez por semana. El consumo de drogas ilícitas (marihuana, cocaína y pasta base) fue definido como su uso al menos una vez al mes.

Al llamado para seleccionar voluntarios acudieron 12 alumnos de ambos sexos, seis de cada colegio, quienes desconocían los objetivos del estudio. Se realizaron cuatro reuniones de capacitación con el grupo de voluntarios. En la primera de ellas se les explicó el objetivo del estudio y la forma de aplicar el instrumento, enfatizando el anonimato y confidencialidad que requeriría la entrevista. En las demás sesiones se practicó la aplicación del instrumento, mediante técnicas de Role-playing y Focus Group.

Para minimizar la posible subdeclaración o sobredeclaración del consumo de drogas, los "pares", cada voluntario, debía entrevistar a alumnos de curso distinto del suyo.

Siguiendo las restricciones de la Metodología de Pares, la entrevista se realizó en los patios, salas y casino de los colegios, donde se tiene acceso a todos los alumnos.

En total, se entrevistaron 124 mujeres y 110 hombres, proviniendo 105 alumnos del colegio municipalizado y los otros 129 del colegio subvencionado. No hubo ningún alumno que se negara a la entrevista. Se solicitó firmar un consentimiento informado tanto a los entrevistadores como a los entrevistados.

Estadística. Los datos fueron analizados con el programa SPSS 14.0. Primero, se determinaron indicadores descriptivos e intervalos de confianza de $95 \%$ y luego, se realizaron pruebas estadísticas bilaterales y unilaterales, con nivel de significación máximo de 5\%, seleccionando cada prueba según la condición de las variables y su distribución. De este modo, se usaron las pruebas de Chi cuadrado, Fisher y prueba $Z$ para estudiar asociación entre variables cualitativas y las pruebas t de Student y Wilcoxon para las variables cuantitativas. 


\section{Anexo I. Instrumento validado: C uestionario sobre el consumo de drogas}

\section{Instrucciones para los entrevistadores}

1. Aclare al entrevistado la privacidad de esta entrevista

2. Obtenga un lugar donde el entrevistado se sienta cómodo

3. Lea claramente las preguntas

4. No interfiera ni aclare dudas al entrevistado

5. Pida al entrevistado que la información que entregue sea veraz y honesta

6. Informe que éste es un proyecto de investigación, cuyo objetivo es conocer la realidad de la magnitud del consumo de drogas

1. Característica sociodemográfica

1.1. Edad: ........... años

1.2. Sexo:
1. mujer
2. hombre

1.3. Carrera o nivel educacional:

1.4. Estado civil
1. soltero
2. casado
3. separado/anulado
4. viudo
5. conviviente

1.5. Comuna de residencia:

2. Consumidor de tabaco: Es aquél que fuma al menos dos veces a la semana

2.1. ¿Has fumado en el último año?
1. sí
2. no

2.2. ¿A qué edad comenzaste a fumar?

2.3. ¿Has sido fumador en los últimos 30 días?
1. sí
2. no

2.4. ¿Cuántos cigarros consumes a la semana (en promedio)?

2.5. ¿Cuántas veces a la semana fumas?

3. Consumidor de alcohol: Consume al menos una vez por semana

3.1. ¿Has consumido alcohol en el último año?
1. sí
2. no

3.2. ¿A qué edad comenzaste a consumir alcohol?

3.3. ¿Has sido un consumidor de alcohol en los últimos 30 días?

1. $s i ́$

2. no
3.4. ¿Cuántos vasos consumes a la semana (en promedio)?

3.5. ¿Cuántas veces a la semana consumes alcohol?

4. Consumidor de marihuana: Consume al menos una vez por semana

4.1. ¿Has consumido marihuana en el último año?

1. sí

2. no

4.2. ¿A qué edad comenzaste a fumar marihuana?

4.3. ¿Has sido un consumidor de marihuana en los últimos 30 días

1. sí

2. no

4.4. ¿Cuántos pitos fumas a la semana (en promedio)?

4.5. ¿Cuántas veces a la semana fumas marihuana?

5. Consumidor de otras drogas: Consume al menos una vez al mes

5.1. ¿Has consumido cocaína en el último año?
1. sí
2. no

5.2. ¿A qué edad comenzaste a consumir cocaína?

5.3. ¿Has sido un consumidor de cocaína en los últimos 30 días?

1. sí

2. no

5.4. ¿Cuántos gramos (líneas) consumes a la semana (en promedio)?

5.5. ¿Cuántas veces al mes consumes cocaína?

6.1. ¿Has tomado éxtasis en el último año?

1. sí

2. no 
6.2. ¿A qué edad comenzaste a consumir éxtasis?

6.3. ¿Has sido un consumidor de éxtasis en los últimos 30 días?

1. sí

2. no

6.4. ¿Cuántas pastillas consumes a la semana (en promedio)?

6.5. ¿Cuántas veces al mes consumes éxtasis?

7.1. ¿Has consumido pasta base en el último año? 1. sí

2. no

7.2. ¿A qué edad comenzaste a consumir?

7.3. ¿Has sido un consumidor de pasta base en los últimos 30 días?

1. sí

2. no

7.4. ¿Cuántos papelillos consumes a la semana (en promedio)?

7.5. ¿Cuántas veces al mes consumes?

8.1. ¿Has consumido en el último año otras drogas?

1. sí ¿cuál?

2. no

8.2. ¿A qué edad la comenzaste a consumir?

8.3. ¿Has sido un consumidor de otras drogas en los últimos 30 días?

1. sí ¿cuál (es)?

2. no

8.4. ¿Cuánto consumes a la semana de la que más consumes (en promedio)?

8.5. ¿Cuántas veces a la semana consumes?
9.1. ¿Has estado en peleas o riñas en el último año? 1. sí

2. no

9.2. ¿Has estado en posesión de algún tipo de armas en estas riñas?

1. sí

2. no

9.3. ¿Cuál (es)?

9.3.1. armas de fuego

9.3.2. cortopunzante

9.3.3. palos u otros elementos

9.4. ¿Has estado en peleas o riñas en los últimos 30 días?

1. Sí

2. no

9.5. ¿Has estado en posesión de algún tipo de armas en estas riñas?

1. Sí

2. no

9.6. ¿Cuál (es)?

9.6.1. armas de fuego

9.6.2. cortopunzante

9.6.3. palos u otros elementos

10.1. ¿Has intentado suicidarte en el último año?
1. sí
2. no

10.2. ¿Has intentado suicidarte en los últimos 30 días?

1. sí

2. no

11.1. ¿Crees en algún dios?

1. sí

2. no

11.2. ¿Cuál es tu religión?

1. católica

2. judía

3. musulmana

4. protestante

5. otra, especificar

\section{Resultados}

Se obtuvo una muestra de 234 escolares, con una edad promedio de 11,5 años y desviación estándar (S) de 1,5 años. El 53\% fueron mujeres, con una edad de 11,6 $\pm 1,5$ años. La edad promedio de los varones fue $11,3 \pm 1,6$ años, sin diferir significativamente de la edad de las niñas $(t=1,27 ; p=0,205)$.
El 62,2\% de los escolares vivía con ambos padres, $24 \%$ con uno de ellos y $13,7 \%$ vivía sin ninguno de sus padres. Cuarenta y ocho coma tres por ciento declaró haber participado en peleas en el último año y 23,1\% haberlo hecho en el último mes. El consumo reciente de tabaco fue $19,5 \%$, de alcohol $17,7 \%$, y drogas lícitas 28,8\%, sin diferencias significativas según sexo en ninguno de dichos grupos (Tabla 1). 


\section{Anexo II. Instrumento adaptado sobre el consumo de tabaco 0 alcohol, para escolares de $4^{\circ}$ a $7^{\circ}$ básico}

\section{Instrucciones para los entrevistadores}

1. Aclare al entrevistado la privacidad de esta entrevista

2. Obtenga un lugar donde el entrevistado se sienta cómodo

3. Lea claramente las preguntas

4. No interfiera ni aclare dudas al entrevistado

5. Pida al entrevistado que la información que entregue sea veraz y honesta

6. Informe que éste es un proyecto de investigación, cuyo objetivo es conocer la realidad de la magnitud del consumo de tabaco y alcohol

1. Característica sociodemográfica

1.1. Edad: ........... años

1.2. Sexo:
1. mujer
2. hombre

1.3. Curso

1.4. ¿Vives con tus padres?
1. con ambos
2. solo con mi mamá
3. solo con mi papá
4. con ninguno
5. con mi mamá y su pareja
6. con mi papá y su pareja
7. otro, especificar

1.5. Comuna de residencia:

2. Consumidor de tabaco: Es aquél que fuma al menos dos veces a la semana

2.1. ¿Has fumado en el último año?
1. sí
2. no

2.2. ¿A qué edad comenzaste a fumar?

2.3. ¿Has sido fumador en los últimos 30 días?
1. sí
2. no

2.4. ¿Cuántos cigarros consumes a la semana (en promedio)?

2.5. ¿Cuántas veces a la semana fumas?

3. Consumidor de alcohol: Consume al menos una vez por semana

3.1. ¿Has consumido alcohol en el último año?
1. sí
2. no

3.2. ¿A qué edad comenzaste a consumir alcohol?
3.3. ¿Has sido un consumidor de alcohol en los últimos 30 días?

1. sí

2. no

3.4. ¿Cuántos vasos consumes a la semana (en promedio)?

3.5. ¿Cuántas veces a la semana consumes alcohol?

3.6. ¿Consumes otro tipo de droga?

1. sí ¿cuáles?

2. no

3.7. ¿Cuántas veces lo has hecho?

3.8. ¿Con quién lo has hecho?

3.9. ¿Sabes de alguien que consuma?
1. Tabaco
SI NO
2. Alcohol
SI NO

4. ¿Sabes si el consumir tabaco es dañino para la salud?

1. sí ¿cuál?

2. no

4.1. ¿Sabes si el consumir alcohol es dañino para la salud?

1. sí ¿̨cuál?

2. no

4.2. ¿Crees en dios?

1. Sí

2. no

4.3. ¿Participas activamente de tus creencias religiosas?

1. Sí

2. no

4.4. ¿Cuántas veces a la semana participas en actividades religiosas? 
Tabla 1. D istribución del consumo anual de drogas lícitas según género

\begin{tabular}{|c|c|c|c|c|c|c|c|}
\hline \multirow[t]{2}{*}{ D rogas lícitas } & \multicolumn{2}{|c|}{ Femenino } & \multicolumn{2}{|c|}{ M asculino } & \multicolumn{2}{|c|}{ Total } & \multirow[t]{2}{*}{ p-value } \\
\hline & $n$ & $\%$ & $n$ & $\%$ & n & $\%$ & \\
\hline \multicolumn{8}{|l|}{ Total $^{1}$} \\
\hline No & 87 & 71,3 & 76 & 71,0 & 163 & 71,2 & 0,481 \\
\hline Sí & 35 & 28,7 & 31 & 29,0 & 66 & 28,8 & 0,481 \\
\hline \multicolumn{8}{|l|}{ Tabaco $^{2}$} \\
\hline No & 99 & 80,5 & 87 & 80,6 & 186 & 80,5 & 0,495 \\
\hline Sí & 24 & 19,5 & 21 & 19,4 & 45 & 19,5 & 0,495 \\
\hline \multicolumn{8}{|l|}{ Alcohol $^{3}$} \\
\hline No & 104 & 84,6 & 87 & 79,8 & 191 & 82,3 & 0,172 \\
\hline Sí & 19 & 15,4 & 22 & 20,2 & 41 & 17,7 & 0,172 \\
\hline
\end{tabular}

Prueba unilateral ${ }^{1} \mathrm{Z}=0,447 ;{ }^{2} \mathrm{Z}=0,000 ;{ }^{3} \mathrm{Z}=0,944$.

El consumo actual de tabaco fue 10,6\%, de alcohol $7,9 \%$ y drogas lícitas fue $14,7 \%$. El consumo de tabaco fue mayor en las mujeres $(13 \% \mathrm{v} / \mathrm{s}$ $7,7 \%$ ), pero sin ser significativo, mientras que el consumo de alcohol fue significativamente mayor en los varones $(11,4 \% \mathrm{v} / \mathrm{s} 4,9 \%)$ (Tabla 2 ).

Al caracterizar el consumo de tabaco, se observó una edad de inicio al consumo promedio de 11 años ( $\pm 1,5$ años), una cantidad media de consumo equivalente a 4,3 cigarillos/semana y una frecuencia media de consumo de 3,1 veces/ semana. $\mathrm{Al}$ analizar dichas variables según género, se observó que los hombres se inician más precozmente $(10,9 \mathrm{v} / \mathrm{s} 11,1$ años) y que fuman mayor cantidad de cigarros (5,5 v/s 3,5 cigarros/ semana) y con mayor frecuencia que las niñas $(3,4$ v/s 2,9 veces/semana), sin embargo ninguna de estas diferencias alcanzó significación estadística (Tabla 3).

Respecto del consumo de alcohol, se encontró una edad de inicio al consumo promedio de 11,3 años ( $\pm 1,8$ años), una cantidad media de consumo de 3,2 vasos/semana y una frecuencia media de consumo de 1,8 veces/semana. La edad de inicio fue similar en ambos géneros $(11,2 \pm 1,3$ años y $11,4 \pm 2,3$ años), mientras que la cantidad y la frecuencia de consumo fueron mayores en el sexo masculino (3,4 v/s 3 vasos/semana y $1,9 \mathrm{v} /$ s 1,6 veces/semana), pero sin ser significativas (Tabla 3).

Tabla 2. D istribución del consumo actual de drogas lícitas según género

\begin{tabular}{|c|c|c|c|c|c|c|c|}
\hline \multirow[t]{2}{*}{ D rogas lícitas } & \multicolumn{2}{|c|}{ Femenino } & \multicolumn{2}{|c|}{ M asculino } & \multicolumn{2}{|c|}{ Total } & \multirow[t]{2}{*}{ p-value } \\
\hline & $\mathbf{n}$ & $\%$ & $\mathbf{n}$ & $\%$ & $\mathbf{n}$ & $\%$ & \\
\hline \multicolumn{8}{|l|}{ Total $^{1}$} \\
\hline No & 104 & 85,2 & 87 & 85,3 & 191 & 85,3 & 0,496 \\
\hline Sí & 18 & 14,8 & 15 & 14,7 & 33 & 14,7 & 0,496 \\
\hline \multicolumn{8}{|l|}{ Tabaco $^{2}$} \\
\hline No & 107 & 87,0 & 96 & 92,3 & 203 & 89,4 & 0,097 \\
\hline Sí & 16 & 13,0 & 8 & 7,7 & 24 & 10,6 & 0,097 \\
\hline \multicolumn{8}{|l|}{ Alcohol $^{3}$} \\
\hline No & 116 & 95,1 & 93 & 88,6 & 209 & 92,1 & 0,035 \\
\hline Sí & 6 & 4,9 & 12 & 11,4 & 18 & 7,9 & 0,035 \\
\hline
\end{tabular}

Prueba unilateral ${ }^{1} \mathrm{Z}=0,00 ;{ }^{2} \mathrm{Z}=1.298 ;{ }^{3} \mathrm{Z}=1,81$. 
Tabla 3. D istribución de la magnitud y frecuencia del consumo de drogas lícitas según género

\begin{tabular}{|c|c|c|c|c|c|c|c|}
\hline \multirow[t]{2}{*}{ Consumo } & \multicolumn{2}{|c|}{ Femenino } & \multicolumn{2}{|c|}{ M asculino } & \multicolumn{2}{|c|}{ Total } & \multirow[t]{2}{*}{ p-value } \\
\hline & $x$ & S & $x$ & $\mathbf{S}$ & $x$ & $\mathbf{S}$ & \\
\hline \multicolumn{8}{|l|}{ Tabaco } \\
\hline Edad de inicio ${ }^{2}$ & 11,1 & 1,3 & 10,9 & 1,9 & 11 & 1,5 & 0,615 \\
\hline Número de veces ${ }^{2}$ & 2,9 & 2,3 & 3,4 & 2,4 & 3,1 & 2,3 & 0,624 \\
\hline Número de cigarros ${ }^{1}$ & 3,5 & 3,1 & 5,5 & 6,8 & 4,3 & 4,8 & 0,353 \\
\hline \multicolumn{8}{|l|}{ Alcohol } \\
\hline Edad de inicio ${ }^{1}$ & 11,2 & 1,3 & 11,4 & 2,3 & 11,3 & 1,8 & 0,735 \\
\hline Número de veces ${ }^{2}$ & 1,6 & 0,5 & 1,9 & 1,6 & 1,8 & 1,3 & 0,519 \\
\hline Número de vasos ${ }^{2}$ & 3,0 & 3,2 & 3,4 & 2,9 & 3,2 & 3,0 & 0,779 \\
\hline
\end{tabular}

${ }^{1} \mathrm{t}$ student (varianzas distintas); ${ }^{2} \mathrm{t}$ student (varianzas iguales).

Al relacionar consumo con supervisión parental, se observó una mayor prevalencia de consumo de drogas lícitas en los escolares que no viven con sus padres $(41,9 \%)$ que en aquellos que viven con ambos padres o sólo con su mamá $(26,1 \%$ y 29,1\%) (Tabla 4).

Se detectó una asociación entre el consumo reciente y actual de drogas lícitas y el consumo de drogas ilícitas, de modo que $100 \%$ de los escolares que consume drogas ilícitas consume además tabaco o alcohol, mientras que en el grupo de escolares que no consume drogas ilícitas, solo $25,7 \%$ consume tabaco o alcohol.

También se encontró una asociación entre el consumo de drogas lícitas y la participación en peleas callejeras, de modo que $40,5 \%$ de los escolares que participaron en peleas callejeras en el último año y $43,4 \%$ de quienes lo hicieron

Tabla 4. Asociación del consumo de drogas lícitas

\begin{tabular}{|c|c|c|c|c|c|c|c|}
\hline \multirow[t]{3}{*}{ C aracterísticas } & \multicolumn{4}{|c|}{ Consumo de drogas } & & & \multirow{3}{*}{ p-value } \\
\hline & \multicolumn{2}{|c|}{ No } & \multicolumn{2}{|c|}{ Sí } & \multicolumn{2}{|c|}{ Total } & \\
\hline & $\mathbf{n}$ & $\%$ & $\mathbf{n}$ & $\%$ & $\mathbf{n}$ & $\%$ & \\
\hline \multicolumn{8}{|l|}{ Vives $\operatorname{con}^{1}$} \\
\hline Ambos padres & 105 & 73,9 & 37 & 26,1 & 142 & 62,3 & 0,210 \\
\hline Sólo mamá & 39 & 70,9 & 16 & 29,1 & 55 & 24,1 & 0,210 \\
\hline Otra & 18 & 58,1 & 13 & 41,9 & 31 & 13,6 & 0,210 \\
\hline \multicolumn{8}{|l|}{ Drogas ilícitas 2} \\
\hline No & 159 & 74,3 & 55 & 25,7 & 214 & 96,4 & 0,000 \\
\hline SÍ & 0 & 0,0 & 8 & 100,0 & 8 & 3,6 & 0,000 \\
\hline \multicolumn{8}{|c|}{ Peleas callejeras año ${ }^{3}$} \\
\hline No & 97 & 82,2 & 21 & 17,8 & 118 & 51,5 & 0,000 \\
\hline SÍ & 66 & 59,5 & 45 & 40,5 & 111 & 48,5 & 0,000 \\
\hline \multicolumn{8}{|c|}{ Peleas callejeras mes ${ }^{4}$} \\
\hline No & 133 & 75,6 & 43 & 24,4 & 176 & 76,9 & 0,007 \\
\hline SÍ & 30 & 56,6 & 23 & 43,4 & 53 & 23,1 & 0,007 \\
\hline
\end{tabular}

Prueba bilateral ${ }^{1} \mathrm{chi}^{2}=3,12 ;{ }^{2}$ Fisher; ${ }^{3} \mathrm{Chi}^{2}=14,422 ;{ }^{4} \mathrm{Chi}^{2}=7,141$. 
en el último mes, son consumidores de drogas lícitas.

La prevalencia de consumo reciente de drogas ilícitas fue de 3,5\%, y se asoció con el consumo actual y reciente de tabaco y de alcohol. Al comparar las características de consumo de tabaco y alcohol entre quienes consumen drogas ilícitas y quienes no, se observó que los consumidores de drogas ilícitas presentaban una edad de inicio al consumo significativamente menor tanto para tabaco (9,2 v/s 11,4) como para el alcohol (10,1 v/s 11,6 años) (Tabla 5). Además presentaron una mayor magnitud de consumo de tabaco ( $6 \mathrm{v} / \mathrm{s} 3,8$ cigarros/semana) y una mayor frecuencia de consumo de alcohol (2,4 v/s 1,5 veces/semana), pero sin alcanzar significancia estadística (Tabla 6).
DisCUSIÓN

Los estudios internacionales muestran que los consumidores de drogas se concentran mayoritariamente entre los 18 y 25 años de edad, rango que en Chile se extiende de los 14 a los 35 años. Ello explica por qué la mayoría de los estudios sobre drogas están orientados a dicho grupo etáreo, siendo muy pocos los que incluyen a los menores de 14 años. Por otra parte, sí sabemos que hay una importante subdeclaración del consumo en todos los grupos etáreos por temor a la marginación o a recibir sanciones, y que según CONACE $^{12}$ la mayor parte de los escolares encuestados señaló que sus padres estarían muy molestos si supieran que su hijo consume drogas,

Tabla 5. Asociación del consumo de drogas ilícitas

\begin{tabular}{|c|c|c|c|c|c|c|c|}
\hline \multirow{3}{*}{ C aracterísticas } & \multicolumn{4}{|c|}{ Consumo de drogas } & & & \multirow{3}{*}{ p-value } \\
\hline & \multicolumn{2}{|c|}{ No } & \multicolumn{2}{|c|}{ Sí } & \multicolumn{2}{|c|}{ Total } & \\
\hline & $\mathbf{n}$ & $\%$ & $\mathbf{n}$ & $\%$ & $\mathbf{n}$ & $\%$ & \\
\hline \multicolumn{8}{|l|}{ Vives $\operatorname{con}^{1}$} \\
\hline Ambos padres & 134 & 95,7 & 6 & 4,3 & 140 & 61,9 & 0,700 \\
\hline Sólo mamá & 54 & 98,2 & 1 & 1,8 & 55 & 24,3 & 0,700 \\
\hline Otra & 30 & 96,8 & 1 & 3,2 & 31 & 13,7 & 0,700 \\
\hline \multicolumn{8}{|c|}{ Peleas callejeras año ${ }^{2}$} \\
\hline No & 116 & 98,3 & 2 & 1,7 & 118 & 52,0 & 0,116 \\
\hline Sí & 103 & 94,5 & 6 & 5,5 & 109 & 48,0 & 0,116 \\
\hline \multicolumn{8}{|c|}{ Peleas callejeras mes ${ }^{2}$} \\
\hline No & 171 & 97,7 & 4 & 2,3 & 175 & 77,1 & 0,083 \\
\hline Sí & 48 & 92,3 & 4 & 7,7 & 52 & 22,9 & 0,083 \\
\hline \multicolumn{8}{|l|}{ Tabaco año ${ }^{2}$} \\
\hline $\mathrm{No}$ & 180 & 98,9 & 2 & 1,1 & 182 & 81,3 & 0,001 \\
\hline Sí & 36 & 85,7 & 6 & 14,3 & 42 & 18,7 & 0,001 \\
\hline \multicolumn{8}{|l|}{ Tabaco mes ${ }^{2}$} \\
\hline No & 195 & 98,5 & 3 & 1,5 & 198 & 90,0 & 0,000 \\
\hline Sí & 17 & 77,3 & 5 & 22,7 & 22 & 10,0 & 0,000 \\
\hline \multicolumn{8}{|l|}{ Alcohol año ${ }^{2}$} \\
\hline $\mathrm{No}$ & 184 & 99,5 & 1 & 0,5 & 185 & 82,2 & 0,000 \\
\hline Sí & 33 & 82,5 & 7 & 17,5 & 40 & 17,8 & 0,000 \\
\hline \multicolumn{8}{|l|}{ Alcohol mes ${ }^{2}$} \\
\hline No & 199 & 98,5 & 3 & 1,5 & 202 & 91,8 & 0,000 \\
\hline Sí & 13 & 72,2 & 5 & 27,8 & 18 & 8,2 & 0,000 \\
\hline
\end{tabular}

${ }^{1}$ chi $=0,715 ;{ }^{2}$ Fisher. 
Tabla 6. Asociación del consumo de drogas ilícitas

\begin{tabular}{|c|c|c|c|c|c|c|c|}
\hline \multirow[t]{3}{*}{ Características } & \multicolumn{7}{|c|}{ Consumo de drogas } \\
\hline & \multicolumn{2}{|c|}{ No } & \multicolumn{2}{|c|}{ Sí } & \multicolumn{3}{|c|}{ Total } \\
\hline & $x$ & $\mathbf{S}$ & $x$ & $\mathbf{S}$ & $\mathbf{x}$ & $\mathbf{S}$ & p-value \\
\hline \multicolumn{8}{|l|}{ Edad de inicio } \\
\hline Tabaco $^{1 \mathrm{a}}$ & 11,4 & 1,4 & 9,2 & 1,2 & 11,1 & 1,4 & 0,0050 \\
\hline Alcohol $^{2 b}$ & 11,6 & 1,9 & 10,1 & 1,2 & 11,2 & 1,8 & 0,0365 \\
\hline \multicolumn{8}{|l|}{ Tabaco } \\
\hline Magnitud ${ }^{3 \mathrm{~b}}$ & 3,8 & 4,6 & 6,0 & 6,0 & 4,3 & 4,8 & 0,287 \\
\hline Frecuencia ${ }^{4 a}$ & 3,2 & 2,6 & 3,0 & 2,0 & 3,1 & 2,3 & 0,832 \\
\hline \multicolumn{8}{|l|}{ Alcohol } \\
\hline Magnitud $^{5 b}$ & 3,5 & 3,4 & 2,9 & 1,5 & 3,2 & 3,0 & 0,639 \\
\hline Frecuencia ${ }^{6 b}$ & 1,5 & 0,7 & 2,4 & 2,1 & 1,8 & 1,3 & 0,121 \\
\hline
\end{tabular}

$a_{t}$ students (varianzas iguales); $b_{t}$ students (varianzas distintas).

${ }^{1} \mathrm{t}=3,649 ;{ }^{2} \mathrm{t}=1,852 ;{ }^{3} \mathrm{t}=-1,082 ;{ }^{4} \mathrm{t}=0,214 ;{ }^{5} \mathrm{t}=0,476 ;{ }^{6} \mathrm{t}=-1,62$.

entonces resulta fácil imaginar que la magnitud del consumo de drogas en escolares, especialmente bajo 14 años, está siendo subvalorada por los estudios tradicionales.

Uno de los principales hallazgos de este estudio fue la precocidad del inicio del consumo, lo cual concuerda con otros estudios que han usado metodologías especiales para poblaciones ocultas $^{15-19}$. La edad media de inicio al consumo de tabaco reportada por la encuesta mundial de tabaquismo en escolares de $7^{\circ}$ básico a $1^{\circ}$ medio fue de 12 años, con algunos casos particulares a $\operatorname{los} 10$ años $^{22}$, mientras que en el presente estudio los encuestados declararon un inicio del consumo a partir de los 7 años para el tabaco (7 a 14 años) y de los 8 años para el alcohol (8 a 14 años). La importancia de los índices de consumo de drogas reportados en este artículo radica en el grupo etáreo que la muestra representa, pues la edad promedio de los escolares que declaró un consumo reciente de tabaco fue de 11 años y para el consumo actual fue de 10,9 años, con edades promedio similares para el consumo de alcohol.

Conforme con la literatura disponible, se encontraron indicadores de frecuencia y cantidad de consumo de drogas lícitas e ilícitas superiores en el género masculino respecto del femenino. Las mujeres presentaron una mayor prevalencia reciente y actual de consumo de tabaco en todas las subpoblaciones con índices superiores a otros estudios realizados sobre el consumo de drogas por género. También resulta llamativa la elevada proporción de escolares que declaró consumir drogas ilícitas (3,5\%), y cómo este subgrupo de escolares presentó una edad promedio de inicio al consumo de tabaco y de alcohol significativamente inferior al grupo de escolares que no consumía drogas ilícitas. Esto indica que el inicio precoz del consumo de drogas lícitas favorecería el consumo de aquellas ilícitas.

Finalmente, este artículo pone en evidencia la necesidad de desarrollar estrategias para prevenir el inicio precoz al consumo de drogas lícitas, que deben ir dirigidas a grupos poblacionales de menor edad que los que hasta ahora se han definido como población diana. Es necesario evaluar los resultados de dichas estrategias mediante una estricta vigilancia con estudios seriados que utilicen la Metodología de Pares u otras metodologías especiales para poblaciones ocultas. 


\section{REFERENCIAS}

1. Florenzano R, Valdés M, Serrano T, Rodríguez J, Roizblatt A. Desarrollo Yoico, Familia y Adolescencia. Rev de Psiquiatría y Salud Mental 2001; 34-40.

2. Florenzano R. El adolescente y sus conductas de riesgo. Ediciones Universidad Católica de Chile. Santiago de Chile, 1998.

3. Nishimura T, Hishinuma E, Else I, Goebert E, Andrade N. Ethnicity and Adolescent Substance Use. Cultural Diversity and Ethnic Minority Psychology 2005; 11: 239-58.

4. Mc Whinter M, Florenzano R. Correlatos psicosociales de la farmacología: Resultados de un estudio en Santiago de Chile. Rev Psiquiátrica 1998; 15: 10-22.

5. Ruangkanchanasetr S, Plitponkarnpim A, Hetrakul P, Kongsakon R. Youth risk behavior survey: Bangkok, Thailand. J Adolescent Health 2005; 227-35.

6. Pillon S, O'Brien B, Piedra K. The relationship between drugs use and risk behaviours in Brazilian university students. Rev Latinoam Enfermagem 2005; 1169-76.

7. Boland M, Fitzpatrick P, Scallan E, Daly L, Herity B, Horgan J, Bourke G. Trends in medical student use of tobacco, alcohol and drugs in an Irish university, 1973-2002. Drug Alcohol Depend 2006; 85: 123-8. Epub 2006 Jun 2.

8. Rodríguez J, Fernández am, Hernández e, Ramírez S. Conductas agresivas, consumo de drogas e intentos de suicidios en jóvenes universitarios. Rev Terapia Psicológica 2006; 63-9.

9. Weiner M, Sussman S, Sun P, Dent C. Explaining the link between violence perpetration, victimization and drug use. Addictive Behaviors 2005; 1261-6.

10. Loas G, Guilbaud O, Pérez-Díaz F, Verrier A, Stephan P, LANG F ET Al. Dependency and suicidality in addictive disorders. Psychiatry Research 2005; 137: 1103-11.

11. Consejo Nacional para el Control de Estupefaciente (CONACE) (1994-2006). Estudio nacional sobre drogas en población general de Chile.

12. Consejo Nacional para el Control de Estupefaciente (CONACE) (1999-2007). Estudio nacional de Drogas en Población escolar de Chile.

13. Santis R, Hayden V, Ruiz S, Anselmo E, Torres R, Pérez DE Los Cobos J ET AL. Implementación de la Entrevista de Acceso Privilegiado para caracterizar consumidores de pasta base de cocaína. Rev Chil NeuroPsiquiatr 2004; 42: 273-80.
14. Santis R, Hidalgo CG, Hayden V, Anselmo E, Ruiz S, RODRÍGUEZ J ET AL. Risk behaviors of out-of-treatment cocaine base paste users and cocaine hydrochloride users: A cohort study by means of privileged access interviewers. Proceedings from the Sixty Sixth Annual Scientific Meeting of the College on Problems of Drug Dependence. San Juan, Puerto Rico. Available at: http://biopsych.com:81/CPDD04_Web/ MeetProgAbSearch_04.html

15. Griffiths P, Gossop M, Powis B, Strang J. Reaching hidden populations of drug users by privileged access interviewers: methodological and practical issues. Addiction 1993; 1617-26.

16. Kuebler D, Hausser D. The Swiss Hidden Population Study: practical and methodological aspects of data collection by privileged access interviewers. Addiction 1997; 325-34.

17. Fountain J, Griffiths P. Synthesis of qualitative research on drug use in European Union: report on an EMCDDA project. European Addiction Research 1999; 4-20.

18. WiebeL W. Identifying and gaining access to hidden populations. NIDA Research Monographs 1990; 98 : 4-11.

19. Rodríguez J, Hernández E, Cumsille MA. Implementación de la metodología de pares para estimar el consumo de drogas lícitas e ilícitas. Rev Chilena de Salud Pública 2005; 20-4.

20. Rodríguez J, Hernández E, Fernández AM. Descripción del consumo de drogas lícitas e ilícitas entre género a través de la metodología de pares. Rev MédChile 2007; 135: 449-59.

21. Rodríguez J, Fernández am, Valdés M, Hernández E, Ramírez S, Román A. A Comparison of the Peers Method and Traditional Methodologies, and risk behaviors in studies of the prevalence of drug consumption in a population in of female, Chilean students. The Spanish Journal of Psychology 2008; 11: 564-72.

22. Encuesta Mundial de Tabaco en Jóvenes. 2000-2003 www.Minsal.cl/ici/destacados/lanzamiento\%20emta.ppt

23. Rodríguez J, Hernández E. Análisis de la confiabilidad y validez de constructo de un instrumento que mide el consumo de drogas lícitas e ilícitas en estudiantes universitarios. Rev Terapia Psicológica 2005; 83-90. 